Discrete Comput Geom 33:687-697 (2005)

DOI: $10.1007 / \mathrm{s} 00454-004-1106-6$

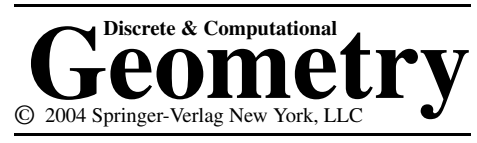

\title{
Monotone Paths in Line Arrangements with a Small Number of Directions
}

\author{
Adrian Dumitrescu \\ Department of Computer Science, University of Wisconsin-Milwaukee, \\ Milwaukee, WI 53201-0784, USA \\ ad@cs.uwm.edu
}

\begin{abstract}
We give subquadratic bounds on the maximum length of an $x$-monotone path in an arrangement of $n$ lines with at most $C \log \log n$ directions, where $C$ is a suitable constant. For instance, the maximum length of an $x$-monotone path in an arrangement of $n$ lines having at most ten slopes is $O\left(n^{67 / 34}\right)$. In particular, we get tight estimates for the case of lines having at most five directions, by showing that previous constructions- $\Omega\left(n^{3 / 2}\right)$ for arrangements with four slopes and $\Omega\left(n^{5 / 3}\right)$ for arrangements with five slopes-due to Sharir and Matoušek, respectively, are (asymptotically) best possible.
\end{abstract}

\section{Introduction}

Consider a set $\mathcal{L}$ of $n$ lines in the plane. The lines of $\mathcal{L}$ induce a cell complex, $\mathcal{A}(\mathcal{L})$, called the arrangement of $\mathcal{L}$, whose vertices are the intersection points of the lines, whose edges are the maximal portions of lines containing no vertices and whose twodimensional cells are the connected components of $\mathbb{R}^{2} \backslash \bigcup_{\ell \in \mathcal{L}} \ell$. It is further assumed that none of the lines is parallel to the $y$-axis. Other than that, parallel or concurrent lines are permitted. One of the properties of a line arrangement with $n$ lines is the maximum possible length, denoted by $\lambda_{n}$, of an $x$-monotone polygonal line (path) composed of edges of the arrangement. The length is defined as the number of turns of the polygonal line plus one (i.e., the number of segments of the polygonal path). See [6] for other properties of line arrangements.

The problem of estimating $\lambda_{n}$ was posed in [4]. An interesting application of this problem can be found in [11]. Sharir established an $\Omega\left(n^{3 / 2}\right)$ lower bound (see [3] and [4]), which was later improved to $\Omega\left(n^{5 / 3}\right)$ by Matoušek [5]. Recently Radoičić and Tóth [9] have raised it to $\Omega\left(n^{7 / 4}\right)$, and very recently, Balogh et al. [1] have brought it up to $\Omega\left(n^{2} / C^{\sqrt{\log n}}\right)$ for some suitable constant $C>1$. 
Clearly the maximum number of vertices in an arrangement of $n$ lines is $\left(\begin{array}{l}n \\ 2\end{array}\right)$, so $\lambda_{n} \leq\left(\begin{array}{l}n \\ 2\end{array}\right)+1=O\left(n^{2}\right)$. A very slight improvement of this bound, which only concerns the constant factor was mentioned in [9]: $\lambda_{n}<5 n^{2} / 12$. This was later improved by Pór to $\lambda_{n}<3 n^{2} / 10$ [8].

As a—perhaps intimately—related problem, Matoušek [5] has also considered arrangements of pseudolines, and longest monotone paths in such arrangements. ${ }^{1}$ For this case, a lower bound of $\Omega\left(n^{2} / \log n\right)$ was given (and obviously the $O\left(n^{2}\right)$ upper bound still holds).

Another related problem is the $k$-level (or its dual, the $k$-set) problem in the plane. The $k$-level of an arrangement of $n$ lines is the closure of the set of points on the lines with the property that there are exactly $k$ lines below them $(k=0, \ldots, n-1)$. The $k$-level of a line arrangement is also a $x$-monotone polygonal path, which turns at each vertex of the arrangement that lies on the path. The $k$-level problem asks for the maximum complexity of the $k$-level in an arrangement of lines. The best upper and lower bounds are both quite recent [2], [10].

In this note we give subquadratic bounds on the maximum length of $x$-monotone paths in arrangements of $n$ lines with at most $C \log \log n$ directions, where $C$ is a suitable constant. In particular, we obtain tight estimates for the case of lines having at most five directions. Theorem 1 below gives a partial answer to the open problem of determining the maximum length of a monotone path in an $n$-line arrangement whose lines have at most $k$ distinct slopes, posed in [1].

Our bounds depend on the well-known Fibonacci numbers, defined by the following recurrence:

$$
F_{0}=1, \quad F_{1}=1, \quad F_{i}=F_{i-1}+F_{i-2}, \quad \text { for } \quad i \geq 2 .
$$

Theorem 1. Let $L_{k}(n)$ be the maximum length of a monotone path in an $n$-line arrangement whose lines have at most $k$ distinct slopes. Then:

(i) $L_{1}(n)=1$.

(ii) $L_{2}(n)=n$.

(iii) $L_{3}(n)=2 n-3$ for $n \geq 4$.

(iv) $L_{4}(n)=\Theta\left(n^{3 / 2}\right)$.

(v) $L_{5}(n)=\Theta\left(n^{5 / 3}\right)$.

(vi) $L_{6}(n)=O\left(n^{9 / 5}\right)$.

(vii) $L_{7}(n)=O\left(n^{15 / 8}\right)$.

(viii) For any $k \geq 4, L_{k}(n) \leq 25 \cdot k \cdot n^{2-1 / F_{k-2}}$, where $F_{k}$ is the $k$ th Fibonacci number.

Remark. The upper bounds in (iv), (v), (vi), and (vii) are special cases of (viii), as is also the example mentioned in the abstract. Note that (viii) also yields (ii) and (iii) up to multiplicative constant factors.

\footnotetext{
${ }^{1}$ A pseudoline is a continuous $x$-monotone curve, and an arrangement of pseudolines is a finite collection of such curves, such that any two meet at at most one point where they cross.
} 

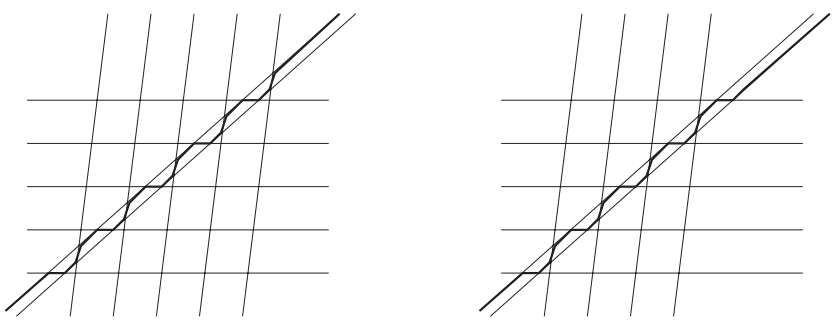

Fig. 1. Arrangement of lines with three slopes which admits a monotone path of length $2 n-3$.

\section{Proof of the Theorem}

The lower bounds in (i) and (ii) are immediate. For (iii), the following construction due to Pálvölgyi [7] gives an exact bound. If $n$ is even, use a bundle of $m$ horizontal lines, a bundle of $m$ near vertical parallel lines of positive slope, so that they form a regular gridlike structure and two (diagonal) lines of slope 1; an example with $m=5$ is illustrated in Fig. 1. If $n$ is odd, add one more horizontal line, see Fig. 1. The path outlined in the figure has length $2 n-3$.

For (iv), we note that the $\Omega\left(n^{3 / 2}\right)$ construction of Sharir mentioned in the Introduction (see [3] and [4]) uses only four slopes. For illustration, we reproduce it here (Fig. 2). For (v), we note that the $\Omega\left(n^{5 / 3}\right)$ construction of Matoušek [5] uses only five slopes. Both constructions can in fact be realized by arrangements of lines in which no two lines are parallel, but they are most easily described in terms of four or five directions.

Now we show the upper bounds. First we introduce some general notation. Consider a set $\mathcal{L}$ of $n$ lines in the plane, with $k$ distinct slopes $s_{1}<\cdots<s_{k}$, and write $n_{i}$ for the number of lines having slope $s_{i}, i=1, \ldots, k$. Denote by $\mathcal{A}$ the corresponding arrangement. For convenience we identify the slopes with their indices. For any subset of slopes $I \subseteq[n]$, consider the subset of lines $\mathcal{L}_{I}$ which consists of all the lines in $\mathcal{L}$ whose slopes are in $I$. Denote by $\mathcal{A}_{I}$ the corresponding arrangement (a sub-arrangement of $\mathcal{A}$ ).

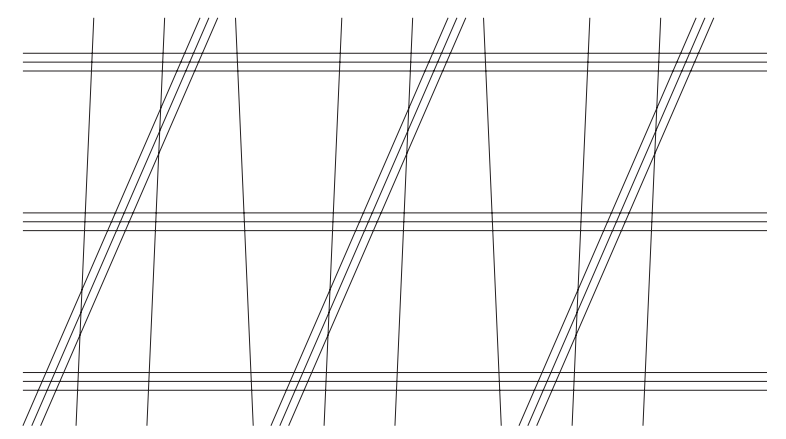

Fig. 2. Arrangement of lines with four slopes which admits a monotone path of length $\Omega\left(n^{3 / 2}\right)$. It consists of: $m$ bundles of $m$ horizontal lines each; $m$ bundles of $m$ lines each at (say) $60^{\circ} ; m(m-1)$ near vertical parallel lines of positive slope, and $m-1$ near vertical parallel lines of negative slope; $m=3$ in this example. 
The following argument will be used repeatedly. Consider the arrangement $\mathcal{A}=$ $\mathcal{A}_{1, \ldots, k}$, and fix a monotone path $p$ in $\mathcal{A}$. Shortcut $p$ to get $p^{\prime}$ a monotone shortcut path in $\mathcal{A}_{1, k}$ in the following way. Replace the portion of $p$ in the interior of each cell of $\mathcal{A}_{1, k}$ by one edge (segment, or half-line for unbounded cells), namely, the segment determined by the endpoints of the portion of $p$ in each cell. Clearly, $p^{\prime}$ is also an $x$-monotone polygonal path (however, it is not a monotone path in $\mathcal{A}_{1, k}$ ). Put $l\left(p^{\prime}\right)$ for its length. It is easy to see that $l\left(p^{\prime}\right)=O(n)$, see Lemma 1 below. A cell of $\mathcal{A}_{1, k}$ is said to be visited by $p$ if $p$ intersects its interior. Put $l(p)$ for the length of $p$, and let $Q\left(\mathcal{A}_{1, k}, p\right)$ be the set of cells of $\mathcal{A}_{1, k}$ which are visited by $p$ and in which $p$ turns. Denote by $l_{c}$ the length of the portion of $p$ inside a cell $c \in Q\left(\mathcal{A}_{1, k}\right)$. Then

$$
l(p) \leq l\left(p^{\prime}\right)+\sum_{c \in Q\left(\mathcal{A}_{1, k}, p\right)}\left(l_{c}-1\right) .
$$

A similar inequality holds if one considers instead a monotone shortcut path in $\mathcal{A}_{1}$ (or $\left.\mathcal{A}_{k}\right)$. In the former case, denote by $Q\left(\mathcal{A}_{1}, p\right)$ the set of cells of $\mathcal{A}_{1}$ which are visited by $p$ and in which $p$ turns. Then

$$
l(p) \leq l\left(p^{\prime}\right)+\sum_{c \in Q\left(\mathcal{A}_{1}, p\right)}\left(l_{c}-1\right) .
$$

Lemma 1. Let $p^{\prime}$ and $p^{\prime \prime}$ be shortcut monotone paths in the arrangements $\mathcal{A}_{1}$ and $\mathcal{A}_{1, k}$, respectively. Put $q^{\prime}=\left|Q\left(\mathcal{A}_{1}, p^{\prime}\right)\right|$ and $q^{\prime \prime}=\left|Q\left(\mathcal{A}_{1, k}, p^{\prime \prime}\right)\right|$. Then:

(i) $l\left(p^{\prime}\right) \leq 2 n_{1}+1$ and $q^{\prime} \leq n_{1}+1$.

(ii) $l\left(p^{\prime \prime}\right) \leq 2 n_{1}+2 n_{k}+1$ and $q^{\prime \prime} \leq n_{1}+n_{k}+1$.

Proof. (i) $p^{\prime}$ cannot revisit any of the lines with slope 1, since the slope of each shortcut edge lies in the open interval $\left(s_{1}, \infty\right)$. By the slope condition, $p^{\prime}$ can have at most two turns on each of the $n_{1}$ lines of minimum slope, which implies the bound on $l\left(p^{\prime}\right)$. The number of cells of $\mathcal{A}_{1}$ is $n_{1}+1$, thus $q^{\prime} \leq n_{1}+1$.

(ii) $p^{\prime \prime}$ cannot revisit any of the lines with slopes 1 or $k$, since the slope of each shortcut edge lies in the open interval $\left(s_{1}, s_{k}\right)$. By the slope condition, $p^{\prime \prime}$ can have at most two turns on each of the $n_{1}+n_{k}$ lines of minimum and maximum slope, which implies the bound on $l\left(p^{\prime \prime}\right)$. The number of cells of $\mathcal{A}_{1, k}$ visited by $p^{\prime \prime}$ is at most $n_{1}+n_{k}+1$, since none of the $n_{1}+n_{k}$ lines of minimum and maximum slope can be revisited by $p^{\prime \prime}$.

Lemma 2. Consider an arrangement $\mathcal{A}=\mathcal{A}_{1, \ldots, k}$ of $n$ lines having $k$ distinct slopes $(k \geq 4)$, and let $p$ be a monotone path in $\mathcal{A}$. Let c be a convex cell $c \in Q\left(\mathcal{A}_{1, k}, p\right)$, and let $p_{c}$ be the portion of $p$ which lies in the interior of $c$. Assume that $\ell_{i}$ and $\ell_{j}$ are two lines of minimum and maximum slope, respectively, which intersect $p_{c}$. Then $\ell_{i}$ and $\ell_{j}$ intersect in the interior of $c$.

Proof. Clearly $p_{c}$ is the contiguous portion of a monotone path in $\mathcal{A}_{2, \ldots, k-1}$ which lies in $c$. Since $p_{c}$ turns in the interior of $c, i \neq j$, thus $2 \leq i<j \leq k-1$. Suppose that the two lines intersect on the boundary of $c$ or outside $c$. We have three cases: 


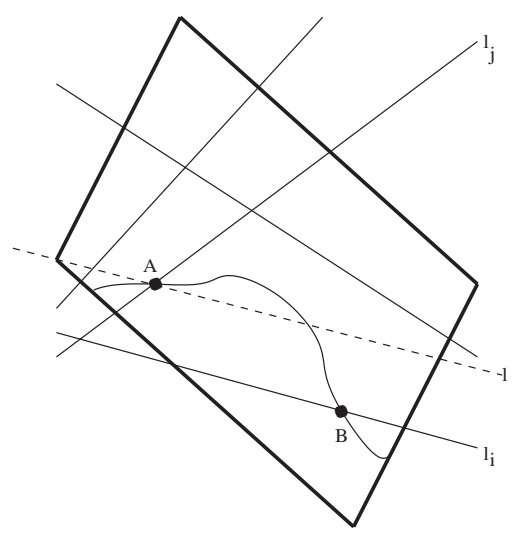

(a)

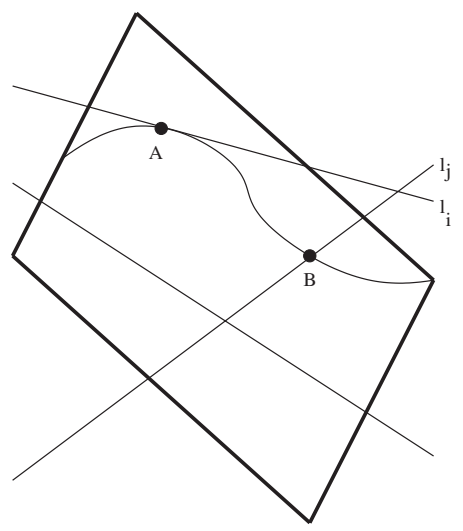

(b)

Fig. 3. Proof of Lemma 2. A cell $c \in Q\left(\mathcal{A}_{1, \ldots, k}, p\right)$, and the portion $p_{c}$ of $p$ which lies in the interior of $c$.

Case 1: $\ell_{i}$ and $\ell_{j}$ intersect before entering $c$-see Fig. 3(a). Assume without loss of generality (flipping the figure gives the other case) that $p_{c}$ intersects $\ell_{j}$ at some point $A$, before intersecting $\ell_{i}$ at some point $B$. Draw a line $\ell$ parallel to $\ell_{i}$ passing through $A$. Since $\ell_{i}$ is a line of minimum slope which intersects $p_{c}$, the portion of $p_{c}$ right of $A$ must lie on or above $\ell$ and thus cannot intersect $\ell_{i}$ inside $c$, which is a contradiction.

Case 2: $\ell_{i}$ and $\ell_{j}$ intersect after leaving $c$-see Fig. 3(b). Assume without loss of generality that $p_{c}$ intersects $\ell_{i}$ at some point $A$, before intersecting $\ell_{j}$ at some point $B$. Since $\ell_{i}$ is a line of minimum slope which intersects $p_{c}$, the portion of $p_{c}$ right of $A$ must lie on or above $\ell_{i}$ and thus cannot intersect $\ell_{j}$ inside $c$, which is a contradiction.

Case 3: The point of intersection of $\ell_{i}$ and $\ell_{j}$ lies on the portion of $\ell_{i}$ left of its intersection with $c$, and on the portion of $\ell_{j}$ right of its intersection with $c$. The argument is similar to that in the previous two cases. (The case when the intersection point lies on the portion of $\ell_{i}$ right of its intersection with $c$, and on the portion of $\ell_{j}$ left of its intersection with $c$ is symmetric).

The argument remains the same for unbounded cells. See also Fig. 4 for an illustration of the case $k=4$.

We return to the proof of Theorem 1 .

(i) Trivially, since all lines are parallel, each monotone path follows exactly one line and the number of turns on any such path is 0 .

(ii) The bound follows from the next lemma.

Lemma 3. Let $m \geq 1$ be the number of vertices in a line arrangement $\mathcal{A}$ of $n$ lines having two distinct slopes, and let $p$ be a monotone path in $\mathcal{A}$. Then $l(p) \leq \min (n, 2 \sqrt{m}+1)$. Furthermore, consider an arrangement of $n$ lines having four distinct slopes, a convex cell $c \in Q\left(\mathcal{A}_{1,4}, p\right)$, and the portion $p_{c}$ of $p$ which lies in the interior of $c$. Then $l\left(p_{c}\right) \leq 2 \sqrt{m_{c}}+1 \leq 3 \sqrt{m_{c}}$, where $m_{c}$ is the number of vertices of $\mathcal{A}_{2,3}$ in the interior of $c$. 


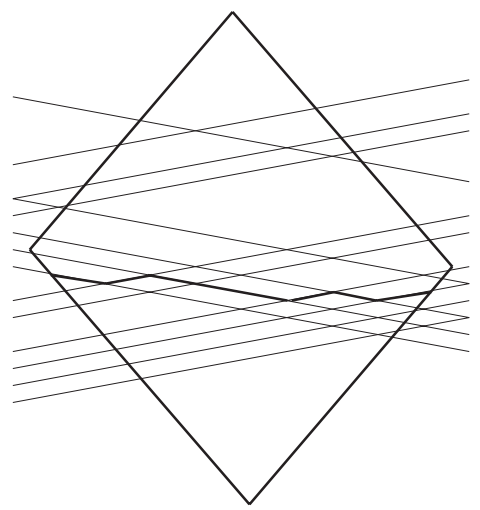

Fig. 4. A cell $c \in Q\left(\mathcal{A}_{1,4}, p\right)$, and the portion $p_{c}$ of $p$ which lies in the interior of $c$. All the $3 \times 4=12$ vertices of the arrangement of the lines in $L_{2} \cup L_{3}$ are in the interior of $c$.

Proof. Write $n=n_{1}+n_{2}$, where $n_{1}$ and $n_{2}$ are the two slope multiplicities. Assume that $n_{1} \leq n_{2}$. Then $n \geq 2 n_{1}$ and $m=n_{1} n_{2} \geq n_{1}^{2}$. Clearly there are at most two turns per line, as no line can be revisited by the path once the path has left that line. Since each turn must involve one of the $n_{1}$ lines, the number of turns is at most $2 n_{1}$. This gives $l(p) \leq 2 n_{1}+1 \leq n+1$. In fact, by charging each turn of the path to the line on which the path goes before the turn, we get that the number of turns is at most $n-1$ (no turn is charged to the line containing the last edge on the path), thus $l(p) \leq n$. We also have $n_{1} \leq \sqrt{m}$, which implies $l(p) \leq 2 n_{1}+1 \leq 2 \sqrt{m}+1$.

For the second part, let $L_{2}$ (resp. $L_{3}$ ) be the set lines of slope 2 (resp. slope 3) which intersect $p_{c}$. Note that $p_{c}$ is the contiguous portion of a monotone path in $\mathcal{A}_{2,3}$ which lies in $c$. Since $c \in Q\left(\mathcal{A}_{1,4}, p\right),\left|L_{2}\right|,\left|L_{3}\right| \geq 1$. By Lemma 2 for $k=4$, all the $\left|L_{2}\right| \cdot\left|L_{3}\right|$ vertices of the arrangement of the lines in $L_{2} \cup L_{3}$ are in the interior of $c$. See Fig. 4. Hence $m_{c} \geq\left|L_{2}\right| \cdot\left|L_{3}\right|$. Assuming $\left|L_{2}\right| \leq\left|L_{3}\right|$, we have $m_{c} \geq\left|L_{2}\right|^{2}$. Thus $l\left(p_{c}\right) \leq 2\left|L_{2}\right|+1 \leq 2 \sqrt{m_{c}}+1 \leq 3 \sqrt{m_{c}}$.

(iii) The bound follows from the next lemma.

Lemma 4. Let $m \geq 1$ be the number of vertices in a line arrangement $\mathcal{A}$ of $n$ lines having three distinct slopes, and let $p$ be a monotone path in $\mathcal{A}$. Then $l(p) \leq \min \left(2 n_{1}+\right.$ $\left.2 n_{3}+1,6 m^{2 / 3}\right)$. Furthermore, consider an arrangement of $n$ lines having five distinct slopes, a convex cell $c \in Q\left(\mathcal{A}_{1,5}, p\right)$, and the portion $p_{c}$ of $p$ which lies in the interior of $c$. Then $l\left(p_{c}\right) \leq 6 m_{c}^{2 / 3}$, where $m_{c}$ is the number of vertices of $\mathcal{A}_{2,3,4}$ in the interior of $c$.

Proof. Let $\mathcal{A}=\mathcal{A}_{1,2,3}$. The lines with minimum and maximum slope (1 and 3) can contribute at most one edge to the path, and thus can contribute at most two turns. On the other hand, each turn on the path must involve either a line of slope 1 or one of slope 3. Consequently,

$$
l(p) \leq 2 n_{1}+2 n_{3}+1 \leq 2 n+1 .
$$

A more careful calculation gives $l(p) \leq 2 n-3$, for $n \geq 4$ (i.e., the bound in Theorem 1): 
If $n_{2}=0, l(p) \leq n \leq 2 n-3$, for $n \geq 4$. If $n_{2}=1$, when the path returns to the diagonal line, it has to go from a "vertical" line to a horizontal line (or the other away around), so instead of $l(p) \leq 2 n_{1}+2 n_{3}+1$, we get $l(p) \leq 3\left(n_{1}+n_{3}\right) / 2+1=(3 n-1) / 2$. It follows that $l(p) \leq 2 n-3$, for $n \geq 4$. Finally, if $n_{2} \geq 2, l(p) \leq 2 n_{1}+2 n_{3}+1 \leq$ $2(n-2)+1=2 n-3$.

Continuing with the proof of the lemma, observe that (3) is a special case of (1). Without loss of generality, we can assume that $n_{1} \leq n_{3}$. Consider the arrangement $\mathcal{A}_{1}$ and use (2) and the bound in Lemma 3. By Lemma $1(\mathrm{i}), l\left(p^{\prime}\right) \leq 2 n_{1}+1 \leq 2 n+1$, and $q=\left|Q\left(\mathcal{A}_{1}, p\right)\right| \leq n_{1}+1 \leq n+1$. We have

$$
l(p) \leq 2 n_{1}+1+\sum_{c \in Q\left(\mathcal{A}_{1}, p\right)}\left(l_{c}-1\right) \leq 2 n_{1}+1+2 \sum_{c \in Q\left(\mathcal{A}_{1}, p\right)} \sqrt{m_{c}} .
$$

Consequently, by Jensen's inequality,

$$
l(p) \leq 2 n_{1}+1+2 q \sqrt{\frac{m}{q}} \leq 2 n_{1}+1+2 \sqrt{n_{1}+1} \sqrt{m} .
$$

We distinguish two cases.

Case 1: $n_{1} \leq m^{1 / 3}$. Inequality (4) implies that

$$
l(p) \leq 2 m^{1 / 3}+1+2\left(m^{1 / 3}+1\right)^{1 / 2} m^{1 / 2} \leq 6 m^{2 / 3} .
$$

Case 2: $n_{1} \geq m^{1 / 3}$. Clearly $m \geq n_{1} n_{3}$. This implies $n_{3} \leq m / m^{1 / 3}=m^{2 / 3}$. Now (3) gives

$$
l(p) \leq 4 m^{2 / 3}+1 \leq 6 m^{2 / 3} .
$$

This proves the first inequality.

The second part follows from a similar argument to that in the proof of Lemma 3. Let $\mathcal{A}=\mathcal{A}_{1,2,3,4,5}$. Note that $p_{c}$ is the contiguous portion of a monotone path in $\mathcal{A}_{2,3,4}$ which lies in $c$. Assume that $L_{i}$ and $L_{j}$ are the set of lines of minimum and maximum slope, respectively, which intersect $p_{c}$. Since $p_{c}$ turns in the interior of $c, i \neq j$ and $\left|L_{i}\right|,\left|L_{j}\right| \geq 1$. By Lemma 2 for $k=5$, all the vertices of the arrangement of the lines in $L_{i} \cup L_{j}$ are in the interior of $c$. Thus $m_{c} \geq\left|L_{i}\right| \cdot\left|L_{j}\right|$, and assume without loss of generality that $\left|L_{i}\right| \leq\left|L_{j}\right|$. Two inequalities, similar to (3) and (4) bound the length of $p_{c}$ :

$$
\begin{aligned}
& l\left(p_{c}\right) \leq 2\left|L_{i}\right|+1+2 \sqrt{\left|L_{i}\right|+1} \sqrt{m_{c}}, \\
& l\left(p_{c}\right) \leq 2\left|L_{i}\right|+2\left|L_{j}\right|+1 .
\end{aligned}
$$

Since $m_{c} \geq\left|L_{i}\right| \cdot\left|L_{j}\right|$, a two-case analysis as in the first part of the proof $\left(\left|L_{i}\right| \leq m_{c}^{1 / 3}\right.$, and $\left|L_{i}\right| \geq m_{c}^{1 / 3}$ ) yields the claimed bound. 
(viii)

Lemma 5. Let $k \geq 2$. Let $m \geq 1$ be the number of vertices in a line arrangement $\mathcal{A}$ of $n$ lines having $k$ distinct slopes, and let p be a monotone path in $\mathcal{A}$. Then

$$
l(p) \leq c_{k} \cdot m^{1-1 / F_{k}}
$$

where

$$
c_{k}=5 \cdot k \cdot 3^{\sum_{i=2}^{k-1}\left(1 / F_{i}\right)},
$$

and $F_{k}$ is the $k$ th Fibonacci number. Furthermore, consider an arrangement of $n$ lines having $k+2$ distinct slopes, a convex cell $c \in Q\left(\mathcal{A}_{1, k+2}, p\right)$, and the portion $p_{c}$ of $p$ which lies in the interior of $c$. Then

$$
l\left(p_{c}\right) \leq c_{k} \cdot m_{c}^{1-1 / F_{k}}
$$

where $m_{c}$ is the number of vertices of $\mathcal{A}_{2, \ldots, k+1}$ in the interior of $c$.

Proof. The argument is similar to the one used in proving Lemmas 3 and 4 . We proceed by induction on $k$. For $k=2$ and $k=3$, the statements follow from Lemmas 3 and 4: $c_{2}=5 \cdot 2=10 \geq 3$ and $c_{3}=5 \cdot 2 \cdot 3^{1 / 2} \geq 6$. Assume now that $k \geq 4$, and the lemma holds for all smaller values of $k^{\prime}, k^{\prime}=2, \ldots, k-1$. Let $\mathcal{A}=\mathcal{A}_{1, \ldots, k}$. Without loss of generality, $n_{1} \leq n_{k}$.

Consider the arrangement $\mathcal{A}_{1}$ and use (2). By Lemma 1(i), $l\left(p^{\prime}\right) \leq 2 n_{1}+1$, and $\left|Q\left(\mathcal{A}_{1}, p\right)\right| \leq n_{1}+1$. The induction hypothesis for $k^{\prime}=k-1$ implies that

$$
l(p) \leq 2 n_{1}+1+\sum_{c \in Q\left(\mathcal{A}_{1}, p\right)}\left(l_{c}-1\right) \leq 2 n_{1}+1+c_{k-1} \sum_{c \in Q\left(\mathcal{A}_{1}, p\right)} m_{c}^{1-1 / F_{k-1}} .
$$

By Jensen's inequality,

$$
l(p) \leq\left(2 n_{1}+1\right)+c_{k-1} \cdot\left(n_{1}+1\right)^{1 / F_{k-1}} \cdot m^{1-1 / F_{k-1}} .
$$

Consider also the arrangement $\mathcal{A}_{1, k}$ and use (1). By Lemma 1(ii), $l\left(p^{\prime \prime}\right) \leq 2 n_{1}+$ $2 n_{k}+1$, and $\left|Q\left(\mathcal{A}_{1, k}, p\right)\right| \leq n_{1}+n_{k}+1$. The induction hypothesis for $k^{\prime}=k-2$ implies that

$$
l(p) \leq\left(2 n_{1}+2 n_{k}+1\right)+c_{k-2} \sum_{c \in Q\left(\mathcal{A}_{1, k}, p\right)} m_{c}^{1-1 / F_{k-2}} .
$$

By Jensen's inequality,

$$
l(p) \leq\left(2 n_{1}+2 n_{k}+1\right)+c_{k-2} \cdot\left(n_{1}+n_{k}+1\right)^{1 / F_{k-2}} \cdot m^{1-1 / F_{k-2}} .
$$

We distinguish two cases:

Case 1: $n_{1} \leq m^{F_{k-2} / F_{k}}$. This implies using (5) that

$$
l(p) \leq\left(2 m^{F_{k-2} / F_{k}}+1\right)+c_{k-1} \cdot\left(m^{F_{k-2} / F_{k}}+1\right)^{1 / F_{k-1}} \cdot m^{1-1 / F_{k-1}} .
$$


Note that

$$
\left(m^{F_{k-2} / F_{k}}+1\right)^{1 / F_{k-1}} \leq 3^{1 / F_{k-1}} \cdot m^{F_{k-2} /\left(F_{k} \cdot F_{k-1}\right)},
$$

since (8) is equivalent to

$$
m^{F_{k-2} / F_{k}}+1 \leq 3 \cdot m^{F_{k-2} / F_{k}}
$$

which is clearly satisfied. So (7) implies that

$$
\begin{aligned}
l(p) & \leq\left(2 m^{F_{k-2} / F_{k}}+1\right)+c_{k-1} \cdot 3^{1 / F_{k-1}} \cdot m^{F_{k-2} /\left(F_{k} \cdot F_{k-1}\right)} \cdot m^{1-1 / F_{k-1}} \\
& \leq 3 \cdot m^{F_{k-2} / F_{k}}+5 \cdot(k-1) \cdot 3^{\sum_{i=2}^{k-2}\left(1 / F_{i}\right)} \cdot 3^{1 / F_{k-1}} \cdot m^{1 / F_{k-1}-1 / F_{k}} \cdot m^{1-1 / F_{k-1}} \\
& =3 \cdot m^{F_{k-2} / F_{k}}+5 \cdot(k-1) \cdot 3^{\sum_{i=2}^{k-1}\left(1 / F_{i}\right)} \cdot m^{1-1 / F_{k}} \\
& \leq 5 \cdot k \cdot 3^{\sum_{i=2}^{k-1}\left(1 / F_{i}\right)} \cdot m^{1-1 / F_{k}} \\
& =c_{k} \cdot m^{1-1 / F_{k}} .
\end{aligned}
$$

Case 2: $n_{1} \geq m^{F_{k-2} / F_{k}}$. Clearly $m \geq n_{1} n_{k}$. This implies $n_{k} \leq m / m^{F_{k-2} / F_{k}}=m^{F_{k-1} / F_{k}}$. Using (6) we have

$$
l(p) \leq\left(4 m^{F_{k-1} / F_{k}}+1\right)+c_{k-2} \cdot\left(2 m^{F_{k-1} / F_{k}}+1\right)^{1 / F_{k-2}} \cdot m^{1-1 / F_{k-2}} .
$$

Note that

$$
\left(2 m^{F_{k-1} / F_{k}}+1\right)^{1 / F_{k-2}} \leq 3^{1 / F_{k-2}} \cdot m^{F_{k-1} /\left(F_{k} \cdot F_{k-2}\right)},
$$

since (11) is equivalent to

$$
2 m^{F_{k-1} / F_{k}}+1 \leq 3 \cdot m^{F_{k-1} / F_{k}},
$$

which clearly holds. So (10) implies that

$$
\begin{aligned}
l(p) & \leq\left(4 m^{F_{k-1} / F_{k}}+1\right)+c_{k-2} \cdot 3^{1 / F_{k-2}} \cdot m^{F_{k-1} /\left(F_{k} \cdot F_{k-2}\right)} \cdot m^{1-1 / F_{k-2}} \\
& \leq 5 \cdot m^{F_{k-1} / F_{k}}+5 \cdot(k-2) \cdot 3^{\sum_{i=2}^{k-3}\left(1 / F_{i}\right)} \cdot 3^{1 / F_{k-2}} \cdot m^{1 / F_{k-2}-1 / F_{k}} \cdot m^{1-1 / F_{k-2}} \\
& =5 \cdot m^{F_{k-1} / F_{k}}+5 \cdot(k-2) \cdot 3^{\sum_{i=2}^{k-2}\left(1 / F_{i}\right)} \cdot m^{1-1 / F_{k}} .
\end{aligned}
$$

Since for $k \geq 4, F_{k-1} \leq F_{k}-1$, we have

$$
\begin{aligned}
l(p) & \leq(5+5(k-2)) \cdot 3^{\sum_{i=2}^{k-2}\left(1 / F_{i}\right)} \cdot m^{1-1 / F_{k}} \\
& \leq 5 \cdot(k-1) \cdot 3^{\sum_{i=2}^{k-1}\left(1 / F_{i}\right)} \cdot m^{1-1 / F_{k}} \\
& \leq 5 \cdot k \cdot 3^{\sum_{i=2}^{k-1}\left(1 / F_{i}\right)} \cdot m^{1-1 / F_{k}} \\
& =c_{k} \cdot m^{1-1 / F_{k}} .
\end{aligned}
$$

This proves the first inequality.

The second part follows from an argument similar to that in the proof of Lemma 3. Let $\mathcal{A}=\mathcal{A}_{1, \ldots, k+2}$. Note that $p_{c}$ is the contiguous portion of a monotone path in $\mathcal{A}_{2, \ldots, k+1}$ which lies in $c$. Assume that $L_{i}$ and $L_{j}$ are the set lines of minimum and maximum slope, respectively, which intersect $p_{c}$. Since $p_{c}$ turns in the interior of $c, i \neq j$ (so 
$2 \leq i<j \leq k+1)$ and $\left|L_{i}\right|,\left|L_{j}\right| \geq 1$. By Lemma 2 , all the vertices of the arrangement of the lines in $L_{i} \cup L_{j}$ are in the interior of $c$. Thus $m_{c} \geq\left|L_{i}\right| \cdot\left|L_{j}\right|$. Assuming without loss of generality that $\left|L_{i}\right| \leq\left|L_{j}\right|$, one can write two inequalities similar to (5) and (6), since by definition, none of the lines in $L_{i}$ or $L_{j}$ can be revisited by $p_{c}$ :

$$
\begin{aligned}
& l\left(p_{c}\right) \leq\left(2\left|L_{i}\right|+1\right)+c_{k-1} \cdot\left(\left|L_{i}\right|+1\right)^{1 / F_{k-1}} \cdot m_{c}^{1-1 / F_{k-1}}, \\
& l\left(p_{c}\right) \leq\left(2\left|L_{i}\right|+2\left|L_{j}\right|+1\right)+c_{k-2} \cdot\left(\left|L_{i}\right|+\left|L_{j}\right|+1\right)^{1 / F_{k-2}} \cdot m_{c}^{1-1 / F_{k-2}} .
\end{aligned}
$$

Since $m_{c} \geq\left|L_{i}\right| \cdot\left|L_{j}\right|$, a two-case analysis as above $\left(\left|L_{i}\right| \leq m_{c}^{F_{k-2} / F_{k}}\right.$, and $\left.\left|L_{i}\right| \geq m_{c}^{F_{k-2} / F_{k}}\right)$ yields the claimed bound.

Corollary 1. For any $k \geq 4, L_{k}(n) \leq 25 \cdot k \cdot n^{2-1 / F_{k-2}}$, where $F_{k}$ is the $k$ th Fibonacci number.

Proof. Let $m \leq\left(\begin{array}{l}n \\ 2\end{array}\right)$ be the number of vertices of the arrangement $\mathcal{A}$, and let $p$ be a monotone path in $\mathcal{A}$. By (6),

$$
l(p) \leq(2 n+1)+5 \cdot 3^{\sum_{i=2}^{\infty}\left(1 / F_{i}\right)} \cdot(k-2) \cdot(n+1)^{1 / F_{k-2}} \cdot\left(\frac{n^{2}}{2}\right)^{1-1 / F_{k-2}} .
$$

Using well-known bounds on the Fibonacci numbers (or by induction), we have

$$
F_{k} \geq\left(\frac{3}{2}\right)^{k} \quad \text { for } \quad k \geq 5
$$

Thus

$$
\sum_{i=2}^{\infty} \frac{1}{F_{i}} \leq \frac{1}{2}+\frac{1}{3}+\frac{1}{5}+\left(\frac{2}{3}\right)^{5} \cdot \sum_{i=0}^{\infty}\left(\frac{2}{3}\right)^{i} \leq 1.43 .
$$

Plugging this bound into (15) yields (using the inequality $F_{k-2} \geq 2$, for $k \geq 4$ )

$$
l(p) \leq 3 n+25 \cdot(k-2) \cdot \frac{2^{1 / F_{k-2}}}{2^{1-1 / F_{k-2}}} \cdot n^{1 / F_{k-2}} \cdot n^{2-2 / F_{k-2}} \leq 25 \cdot k \cdot n^{2-1 / F_{k-2}} .
$$

Corollary 2. There exists an absolute constant $C>0$, so that if $k \leq C \log \log n$, then $L_{k}(n)=o\left(n^{2}\right)$.

Proof. From well-known bounds on the Fibonacci numbers (or by induction), we have

$$
F_{k} \leq\left(\frac{5}{3}\right)^{k} \quad \text { for } \quad k \geq 1
$$

and the corollary follows. 


\section{Concluding Remarks}

Possibly, the factor $k$ in Theorem 1(viii) could be removed by a more careful calculation. However, this would not extend the range of $k$-in terms of $n$-for which Corollary 2 gives a subquadratic bound.

Most likely, the Fibonacci numbers present in the exponents of our bounds have little to do with the right order of magnitude of $L_{k}(n)$ for small values of $k$, and their presence is probably only an artifact of our proofs. For example, one can check that the $\Omega\left(n^{7 / 4}\right)$ construction of Radoičić and Tóth can be realized using only six slopes-thus $L_{6}(n)=\Omega\left(n^{7 / 4}\right)$-while our upper bound is $O\left(n^{9 / 5}\right)$. This is a possible start point if one wants to establish better bounds for small values of $k$. Similarly, for fixed $k$, the recent construction due to Balogh et al. gives $L_{k}(n)=\Omega\left(n^{2-2 / k}\right)$.

Of course, the most interesting question is to give a subquadratic upper bound for an arbitrary number of directions (as is generally believed to hold). Which of the ideas from our note (if any) may be useful for this purpose, remains to be seen.

\section{Acknowledgement}

The author is grateful to William Steiger for suggesting this restricted version of the problem.

\section{References}

1. J. Balogh, O. Regev, C. Smyth, W. Steiger and M. Szegedy, Long monotone paths in line arrangements, Proceedings of the Nineteenth Annual ACM Symposium on Computational Geometry, June 2003, pp. 124-128.

2. T. K. Dey, Improved bounds for planar $k$-sets and related problems, Discrete \& Computational Geometry, 19 (1998), 373-382.

3. H. Edelsbrunner, Algorithms in Combinatorial Geometry, Springer-Verlag, Heidelberg, 1987.

4. H. Edelsbrunner and L. Guibas, Topologically sweeping an arrangement, Journal of Computer and System Sciences, 38 (1989), 165-194.

5. J. J. Matoušek, Lower bounds on the length of monotone paths in arrangements, Discrete \& Computational Geometry, 6 (1991), 129-134.

6. J. Pach and P. K. Agarwal, Combinatorial Geometry, Wiley, New York, 1995.

7. D. Pálvölgyi, Personal communication, 2003.

8. A. Pór, Unpublished manuscript, 2002.

9. R. Radoičić and G. Tóth, Monotone paths in line arrangements, Proceedings of the Seventeenth Annual ACM Symposium on Computational Geometry, 2001, pp. 312-314.

10. G. Tóth, Point sets with many $k$-sets, Discrete \& Computational Geometry, 26 (2001), 187-194.

11. P. Yamamoto, K. Kato, K. Imai, and H. Imai, Algorithms for vertical and orthogonal $L_{1}$ linear approximation of points, Proceedings of the Fourth Annual ACM Symposium on Computational Geometry, 1988, pp. 352-361.

Received October 10, 2003, and in revised form February 24, 2004. Online publication June 1, 2004. 